\title{
A generalized $\theta$-contraction and related fixed point theorems
}

\author{
Mohamed Jleli, Bessem Samet* \\ Department of Mathematics, College of Science King Saud University, P. O. Box 2455, Riyadh 11451, Saudi Arabia. \\ Communicated by $\mathrm{C}$. Vetro
}

\begin{abstract}
We introduce two types of generalized $\theta$-contraction mappings in complete metric spaces. For each type, we study the existence of fixed points. The obtained results in this paper generalize several existing fixed point theorems in the literature. We end this work with some open questions. (C)2017 All rights reserved.
\end{abstract}

Keywords: Fixed point, generalized $\theta$-contraction of type (I), generalized $\theta$-contraction of type (II), partial order, $\theta$-cyclic contraction.

2010 MSC: 47H10.

\section{Introduction}

One of the most important fixed point theorems is the Banach contraction principle [1], which is a well-known tool in solving several existing problems in nonlinear analysis. Many generalizations and extensions of this famous principle exist in the literature. In some works, the contraction nature of the operator is weakened; see $[3,6,8,10,12,13,19,23,25,26,28]$ and references therein. In other works, the topology is weakened; see $[4,5,9,11,14,15,18,27]$ and references therein. In [21], Nadler extended the Banach contraction principle to the case of multi-valued mappings. For fixed point theorems for multi-valued mappings, see $[2,7,17,20,29]$ and references therein.

Let $\Theta$ be the set of functions $\theta:(0, \infty) \rightarrow(1, \infty)$ satisfying the following conditions:

(i) $\theta$ is non-decreasing;

(ii) for each sequence $\left\{t_{n}\right\} \subset(0, \infty), \lim _{n \rightarrow \infty} \theta\left(t_{n}\right)=1$ if and only if $\lim _{n \rightarrow \infty} t_{n}=0^{+}$;

(iii) there exist $r \in(0,1)$ and $\ell \in(0, \infty]$ such that $\lim _{t \rightarrow 0^{+}} \frac{\theta(t)-1}{t^{r}}=\ell$.

Let $\Theta_{c}$ be the set defined by

$$
\Theta_{c}=\{\theta \in \Theta: \theta \text { is continous }\} .
$$

Let $(X, d)$ be a metric space and $T: X \rightarrow X$ be a given mapping. We say that $T$ is a $\theta$-contraction for some $\theta \in \Theta$, if the following condition holds:

$$
x, y \in X, d(T x, T y) \neq 0 \Longrightarrow \theta(d(T x, T y)) \leqslant[\theta(d(x, y))]^{k}
$$

for some constant $k \in(0,1)$. Recently, in [13] Jleli and Samet established the following fixed point theorem.

\footnotetext{
*Corresponding author

Email addresses: jleli@ksu.edu.sa (Mohamed Jleli), bsamet@ksu.edu.sa (Bessem Samet)
} 
Theorem 1.1. Let $(X, \mathrm{~d})$ be a complete metric space and $\mathrm{T}: \mathrm{X} \rightarrow \mathrm{X}$ be a $\theta$-contraction for some $\theta \in \Theta$. Then $\mathrm{T}$ has a unique fixed point.

Observe that any Banach contraction is a $\theta$-contraction for $\theta(t)=e^{t}$. Then Theorem 1.1 is a generalization of the Banach contraction principle [1].

In [12], Jleli et al. established the following extension of Theorem 1.1.

Theorem 1.2. Let $(X, d)$ be a complete metric space and $\mathrm{T}: \mathrm{X} \rightarrow \mathrm{X}$ be a mapping satisfying the following condition:

$$
x, y \in X, d(T x, T y) \neq 0 \Longrightarrow \theta(d(T x, T y)) \leqslant[\theta(\max \{d(x, y), d(x, T x), d(y, T y)\})]^{k}
$$

for some $\theta \in \Theta_{c}$ and $\mathrm{k} \in(0,1)$. Then $\mathrm{T}$ has a unique fixed point.

Our aim in this paper is to establish new extensions of Theorem 1.1 for new classes of mappings. The obtained results in this paper extend and generalize some fixed point theorems from the literature including the Banach contraction principle, Das-Gupta fixed point theorem [8], Jaggi fixed point theorem [10], Ran-Reurings fixed point theorem [24], Nieto-Lopez fixed point theorem [22], and Kirk et al. fixed point theorem for cyclic mappings [16].

\section{Main results}

Let $(X, d)$ be a complete metric space and $T: X \rightarrow X$ be a given mapping. We say that $T$ is a generalized $\theta$-contraction of type (I) for some $\theta \in \Theta_{c}$, if it satisfies the following condition:

$$
x, y \in X, d(T x, T y) \neq 0 \Longrightarrow \theta(d(T x, T y)) \leqslant[\theta(M(x, y))]^{k}
$$

for some $k \in(0,1)$, where

$$
M(x, y)=\max \left\{d(x, y), \frac{d(y, T y)(1+d(x, T x))}{1+d(x, y)}\right\}, x, y \in X
$$

We say that $\mathrm{T}$ is a generalized $\theta$-contraction of type (II) for some $\theta \in \Theta_{c}$, if it satisfies the following condition:

$$
x, y \in X, d(T x, T y) \neq 0 \Longrightarrow \theta(d(T x, T y)) \leqslant[\theta(N(x, y))]^{k},
$$

for some $k \in(0,1)$, where

$$
N(x, y)=\max \left\{d(x, y), \frac{d(y, T y) d(x, T x)}{d(x, y)}\right\}, x, y \in X, x \neq y .
$$

Our first main result in this paper is given by the following theorem.

Theorem 2.1. If $\mathrm{T}: \mathrm{X} \rightarrow \mathrm{X}$ is a generalized $\theta$-contraction of type (I) for some $\theta \in \Theta_{c}$, then it admits a unique fixed point.

Proof. Let $\chi \in X$ be an arbitrary point in $X$. If for some $p \in \mathbb{N}$ we have $T^{p} \chi=T^{p+1} \chi$, then $T^{p} \chi$ will be a fixed point of $T$. So, without restriction of the generality, we can suppose that $d\left(T^{n} x, T^{n+1} \chi\right)>0$ for all $n=0,1, \ldots$ Using (2.1), for every $n=1,2, \ldots$, we obtain

$$
\theta\left(M\left(d\left(T^{n} x, T^{n+1} x\right)\right)\right) \leqslant\left[\theta\left(M\left(T^{n-1} x, T^{n} x\right)\right)\right]^{k},
$$

where

$$
M\left(T^{n-1} x, T^{n} x\right)=\max \left\{d\left(T^{n-1} x, T^{n} x\right), \frac{d\left(T^{n} x, T^{n+1} \chi\right)\left(1+d\left(T^{n-1} \chi, T^{n} \chi\right)\right)}{1+d\left(T^{n-1} \chi, T^{n} x\right)}\right\}
$$




$$
=\max \left\{d\left(T^{n-1} x, T^{n} x\right), d\left(T^{n} x, T^{n+1} x\right)\right\} .
$$

If $M\left(T^{n-1} \chi, T^{n} \chi\right)=d\left(T^{n} \chi, T^{n+1} \chi\right)$, then (2.3) turns into

$$
\theta\left(d\left(T^{n} \chi, T^{n+1} \chi\right)\right) \leqslant\left[\theta\left(T^{n} \chi, T^{n+1} \chi\right)\right]^{k},
$$

which is a contradiction with $k \in(0,1)$. Then we have $M\left(T^{n-1} \chi, T^{n} x\right)=d\left(T^{n-1} \chi, T^{n} \chi\right)$, which yields

$$
\theta\left(d\left(T^{n} \chi, T^{n+1} \chi\right)\right) \leqslant\left[\theta\left(d\left(T^{n-1} \chi, T^{n} \chi\right)\right)\right]^{k} \leqslant\left[\theta\left(d\left(T^{n-2} \chi, T^{n-1} \chi\right)\right)\right]^{k^{2}} \leqslant \cdots \leqslant[\theta(d(x, T \chi))]^{k^{n}} .
$$

Thus we have

$$
1 \leqslant \theta\left(d\left(T^{n} x, T^{n+1} x\right)\right) \leqslant[\theta(d(x, T x))]^{k^{n}}, n=0,1 \ldots
$$

Passing the limit as $n \rightarrow \infty$ in the above inequality, we obtain

$$
\lim _{n \rightarrow \infty} \theta\left(d\left(T^{n} x, T^{n+1} x\right)\right)=1,
$$

which yields

$$
\lim _{n \rightarrow \infty} d\left(T^{n} x, T^{n+1} x\right)=0 .
$$

As consequence, there exist $r \in(0,1)$ and $\ell \in(0, \infty]$ such that

$$
\lim _{n \rightarrow \infty} \frac{\theta\left(d\left(T^{n} x, T^{n+1} \chi\right)\right)-1}{d\left(T^{n} x, T^{n+1} \chi\right)^{r}}=\ell
$$

We distinguish two cases.

Case 1. If $0<\ell<\infty$.

By the definition of the limit, there exists some natural number $n_{0}$ such that

$$
\frac{\theta\left(d\left(T^{n} x, T^{n+1} \chi\right)\right)-1}{d\left(T^{n} x, T^{n+1} \chi\right)^{r}} \geqslant \frac{\ell}{2} \text { for all } n \geqslant n_{0}
$$

which yields

$$
n\left[d\left(T^{n} \chi, T^{n+1} \chi\right)\right]^{r} \leqslant \frac{2}{\ell} n\left[\theta\left(d\left(T^{n} \chi, T^{n+1} \chi\right)\right)-1\right] \text { for all } n \geqslant n_{0} \text {. }
$$

Case 2. If $\ell=\infty$.

Let $B>0$ be an arbitrary positive number. From the definition of the limit, there exists some natural number $\mathrm{n}_{0}$ such that

$$
\frac{\theta\left(d\left(T^{n} \chi, T^{n+1} \chi\right)\right)-1}{d\left(T^{n} \chi, T^{n+1} \chi\right)^{r}} \geqslant B \text { for all } n \geqslant n_{0}
$$

which yields

$$
n\left[d\left(T^{n} \chi, T^{n+1} \chi\right)\right]^{r} \leqslant \frac{1}{B} n\left[\theta\left(d\left(T^{n} \chi, T^{n+1} \chi\right)\right)-1\right] \text { for all } n \geqslant n_{0} .
$$

As consequence, in all cases, there exist $A>0$ and a natural number $n_{0}$ such that

$$
n\left[d\left(T^{n} \chi, T^{n+1} \chi\right)\right]^{r} \leqslant A n\left[\theta\left(d\left(T^{n} \chi, T^{n+1} \chi\right)\right)-1\right] \text { for all } n \geqslant n_{0} .
$$

Using (2.4), we obtain

$$
n\left[d\left(T^{n} x, T^{n+1} x\right)\right]^{r} \leqslant A n\left(\left[\theta\left(d\left(x, T^{n} x\right)\right)\right]^{k^{n}}-1\right) \text { for all } n \geqslant n_{0} .
$$


Passing $n \rightarrow \infty$ in the above inequality, we get

$$
\lim _{n \rightarrow \infty} n\left[d\left(T^{n} x, T^{n+1} x\right)\right]^{r}=0
$$

Then

$$
d\left(T^{n} x, T^{n+1} x\right) \leqslant \frac{1}{n^{1 / r}} \text { for all } n \geqslant n_{1}
$$

for some natural number $n_{1}$. Now, for all $m=1,2, \ldots$ and $n=n_{1}, n_{1}+1, \ldots$, using (2.5), we have

$$
d\left(T^{n} x, T^{n+m} x\right) \leqslant \sum_{i=n}^{n+m-1} d\left(T^{i} x, T^{i+1} \chi\right) \leqslant \sum_{i=n}^{\infty} \frac{1}{i^{1 / r}} .
$$

From the convergence of the series $\sum_{i} \frac{1}{i^{1 / r}}$, we deduce that $\left\{T^{n} \chi\right\}$ is a Cauchy sequence in the complete metric space $(X, d)$. Then, there exists some $z \in X$ such that $\left\{T^{n} \chi\right\}$ converges to $z$. Without restriction of the generality, we can suppose that $T^{n} x \neq z$ for all $n$ (or for $n$ large enough). Suppose that $z$ is not a fixed point of T. Using (2.1), we have

$$
\theta\left(M\left(d\left(T^{n+1} \chi, T z\right)\right)\right) \leqslant\left[\theta\left(M\left(T^{n} \chi, z\right)\right)\right]^{k} \text { for all } n,
$$

where

$$
M\left(T^{n} \chi, z\right)=\max \left\{d\left(T^{n} \chi, z\right), \frac{d(z, T z)\left(1+d\left(T^{n} \chi, T^{n+1} \chi\right)\right)}{1+d\left(T^{n} \chi, z\right)}\right\} .
$$

Letting $n \rightarrow \infty$ in the above inequality and using the continuity of $\theta$, we obtain

$$
\theta(d(z, T z)) \leqslant[\theta(d(z, T z))]^{k}<\theta(d(z, T z)),
$$

which is a contradiction. Thus we proved that $z$ is a fixed point of $\mathrm{T}$. Suppose now that $w \in X$ is another fixed point of T. From (2.1), we have

$$
\theta(d(z, w))=\theta(d(T z, T w)) \leqslant[\theta(M(z, w))]^{k},
$$

where

$$
M(z, w)=\max \left\{d(z, w), d(w, T w) \frac{1+d(z, T z}{1+d(z, w)}\right\}=d(z, w)
$$

Then

$$
\theta(d(z, w)) \leqslant[\theta(d(z, w))]^{k}<\theta(d(z, w)),
$$

which is a contradiction. As consequence, we have one and only one fixed point.

We deduce now the following fixed point theorem.

Corollary 2.2. Suppose that $\mathrm{T}: \mathrm{X} \rightarrow \mathrm{X}$ satisfies the following condition: for all $\mathrm{x}, \mathrm{y} \in \mathrm{X}$,

$$
d(T x, T y) \leqslant k \max \left\{d(x, y), \frac{d(y, T y)(1+d(x, T x))}{1+d(x, y)}\right\}
$$

for some $\mathrm{k} \in(0,1)$. Then $\mathrm{T}$ has a unique fixed point.

Proof. We observe easily that $T$ is a generalized $\theta$-contraction of type (I) with $\theta(t)=e^{\sqrt{t}}$. Then the result follows immediately from Theorem 2.1.

An immediate consequence of the above result is the following fixed point theorem due to Das and Gupta [8]. 
Corollary 2.3. Suppose that $\mathrm{T}: \mathrm{X} \rightarrow \mathrm{X}$ satisfies the following condition: for all $\mathrm{x}, \mathrm{y} \in \mathrm{X}$,

$$
d(T x, T y) \leqslant \alpha d(x, y)+\beta \frac{d(y, T y)(1+d(x, T x))}{1+d(x, y)}
$$

for some $\alpha, \beta \geqslant 0$ such that $0<\alpha+\beta<1$. Then $\mathrm{T}$ has a unique fixed point.

Take $\beta=0$ in the above result, we obtain the Banach contraction principle [1].

Our second main result is given by the following theorem.

Theorem 2.4. If $\mathrm{T}: \mathrm{X} \rightarrow \mathrm{X}$ is a generalized $\theta$-contraction of type (II) for some $\theta \in \Theta_{c}$, then it admits a unique fixed point.

Proof. Let $\chi \in X$ be an arbitrary point in $X$. If for some $p \in \mathbb{N}$ we have $T^{p} \chi=T^{p+1} \chi$, then $T^{p} \chi$ will be a fixed point of $T$. So, without restriction of the generality, we can suppose that $d\left(T^{n} \chi, T^{n+1} \chi\right)>0$ for all $n=0,1, \ldots$ Using (2.2), for every $n=1,2, \ldots$, we obtain

$$
\theta\left(d\left(T^{n} x, T^{n+1} x\right)\right) \leqslant\left[\theta\left(N\left(T^{n-1} x, T^{n} x\right)\right)\right]^{k},
$$

where

$$
\begin{aligned}
N\left(T^{n-1} x, T^{n} x\right) & =\max \left\{d\left(T^{n-1} x, T^{n} x\right), \frac{d\left(T^{n} x, T^{n+1} x\right) d\left(T^{n-1} x, T^{n} x\right)}{d\left(T^{n-1} x, T^{n} x\right)}\right\} \\
& =\max \left\{d\left(T^{n-1} x, T^{n} x\right), d\left(T^{n} x, T^{n+1} x\right)\right\} .
\end{aligned}
$$

The rest of the proof follows the same lines as the proof of Theorem 1.1.

Corollary 2.5. Suppose that $\mathrm{T}: \mathrm{X} \rightarrow \mathrm{X}$ satisfies the following condition: for all $\mathrm{x}, \mathrm{y} \in \mathrm{X}$ with $\mathrm{x} \neq \mathrm{y}$

$$
d(T x, T y) \leqslant k \max \left\{d(x, y), \frac{d(y, T y) d(x, T x)}{d(x, y)}\right\}
$$

for some $\mathrm{k} \in(0,1)$. Then $\mathrm{T}$ has a unique fixed point.

Proof. The mapping T is a generalized $\theta$-contraction of type (II) with $\theta(t)=e^{\sqrt{t}}$. Then the result follows from Theorem 2.4.

From the above result, we deduce the following fixed point theorem due to Jaggi [10].

Corollary 2.6. Suppose that $\mathrm{T}: \mathrm{X} \rightarrow \mathrm{X}$ satisfies the following condition: for all $\mathrm{x}, \mathrm{y} \in \mathrm{X}$ with $\mathrm{x} \neq \mathrm{y}$,

$$
d(T x, T y) \leqslant \alpha d(x, y)+\beta \frac{d(y, T y) d(x, T x)}{d(x, y)}
$$

for some $\alpha, \beta \geqslant 0$ such that $0<\alpha+\beta<1$. Then $\mathrm{T}$ has a unique fixed point.

Let $\preceq$ be a partial order on $X$. Set

$$
E_{\preceq}=\{(x, y) \in X \times X: x \preceq y \text { or } y \preceq x\} .
$$

We say that $\mathrm{T}: \mathrm{X} \rightarrow \mathrm{X}$ preserves order if

$$
(x, y) \in E_{\preceq} \Rightarrow(T x, T y) \in E_{\preceq} .
$$

We say that $x_{0} \in X$ is a starting point if $\left(x_{0}, T x_{0}\right) \in E_{\preceq}$. 
We say that $X$ is $E \preceq$-regular if for every sequence $\left\{x_{n}\right\} \subset X$ such that $\left(x_{n}, x_{n+1}\right) \in E_{\preceq}$ for every $n$, we have

$$
x_{n} \rightarrow x \text { as } n \rightarrow \infty \Rightarrow\left(x_{n}, x\right) \in E_{\preceq} \text { for every } n .
$$

We say that $T$ is a generalized $\preceq-\theta$-contraction of type (I) for some $\theta \in \Theta$, if it satisfies the following condition:

$$
(x, y) \in E_{\preceq}, d(T x, T y) \neq 0 \Longrightarrow \theta(d(T x, T y)) \leqslant[\theta(M(x, y))]^{k}
$$

for some $k \in(0,1)$

We say that $T$ is a generalized $\preceq$ - $\theta$-contraction of type (II) for some $\theta \in \Theta$, if it satisfies the following condition:

$$
(x, y) \in E_{\preceq}, d(T x, T y) \neq 0 \Longrightarrow \theta(d(T x, T y)) \leqslant[\theta(N(x, y))]^{k}
$$

for some $k \in(0,1)$.

We have the following result.

Theorem 2.7. Let $\mathrm{T}: \mathrm{X} \rightarrow \mathrm{X}$ be a generalized $\preceq$ - $\theta$-contraction of type (I) for some $\theta \in \Theta$. Suppose that $\mathrm{T}$ is continuous and it preserves order. If there exists a starting point $x_{0} \in X$, then $T$ has a fixed point.

Proof. Since $x_{0} \in X$ is a starting point and the operator $T$ preserves order, then

$$
\left(T^{n} x_{0}, T^{n+1} \chi_{0}\right) \in E_{\preceq} \text { for all } n \text {. }
$$

Let $\left\{x_{n}\right\} \subset X$ be the sequence defined by

$$
x_{n}=T^{n} x_{0} \text { for all } n
$$

Without restriction of the generality, we may suppose that $x_{n} \neq x_{n+1}$ for every $n$. Otherwise, the existence of a fixed point is immediate. Now, we argue by a similar way as in the proof of Theorem 2.1 to see that $\left\{x_{n}\right\}$ is a Cauchy sequence in the complete metric space $(X, d)$. Let $z \in X$ be such that $x_{n} \rightarrow z$ as $n \rightarrow \infty$. The continuity of $T$ yields $T z=z$, that is, $z$ is a fixed point of $T$.

The following results are immediate consequences of Theorem 2.7.

Corollary 2.8. Suppose that $\mathrm{T}: \mathrm{X} \rightarrow \mathrm{X}$ satisfies the following condition: for all $(\mathrm{x}, \mathrm{y}) \in \mathrm{E}_{\preceq}$,

$$
d(T x, T y) \leqslant k \max \left\{d(x, y), \frac{d(y, T y)(1+d(x, T x))}{1+d(x, y)}\right\}
$$

for some $\mathrm{k} \in(0,1)$. If $\mathrm{T}$ preserves order and there exists a starting point $\mathrm{x}_{0} \in \mathrm{X}$, then $\mathrm{T}$ has a fixed point.

Corollary 2.9. Suppose that $\mathrm{T}: \mathrm{X} \rightarrow \mathrm{X}$ satisfies the following condition: for all $(x, y) \in \mathrm{E}_{\preceq}$,

$$
d(T x, T y) \leqslant \alpha d(x, y)+\beta \frac{d(y, T y)(1+d(x, T x))}{1+d(x, y)}
$$

for some $\alpha, \beta \geqslant 0$ such that $0<\alpha+\beta<1$. If $\mathrm{T}$ preserves order and there exists a starting point $\mathrm{x}_{0} \in \mathrm{X}$, then $\mathrm{T}$ has a fixed point.

Take $\beta=0$ in the above result, we obtain Ran-Reurings fixed point theorem [24].

Using similar arguments as in the proof of Theorem 2.7, we can establish the following result.

Theorem 2.10. Let $\mathrm{T}: X \rightarrow X$ be a generalized $\preceq-\theta$-contraction of type (II) for some $\theta \in \Theta$. Suppose that $\mathrm{T}$ is continuous and it preserves order. If there exists a starting point $x_{0} \in X$, then $\mathrm{T}$ has a fixed point.

As consequences, we deduce the following results. 
Corollary 2.11. Suppose that $\mathrm{T}: \mathrm{X} \rightarrow \mathrm{X}$ satisfies the following condition: for all $(\mathrm{x}, \mathrm{y}) \in \mathrm{E}_{\preceq}$ with $\mathrm{x} \neq \mathrm{y}$,

$$
d(T x, T y) \leqslant k \max \left\{d(x, y), \frac{d(y, T y) d(x, T x)}{d(x, y)}\right\}
$$

for some $\mathrm{k} \in(0,1)$. If $\mathrm{T}$ preserves order and there exists a starting point $\mathrm{x}_{0} \in \mathrm{X}$, then $\mathrm{T}$ has a fixed point.

Corollary 2.12. Suppose that $\mathrm{T}: \mathrm{X} \rightarrow \mathrm{X}$ satisfies the following condition: for all $(\mathrm{x}, \mathrm{y}) \in \mathrm{E}_{\preceq}$ with $\mathrm{x} \neq \mathrm{y}$,

$$
d(T x, T y) \leqslant \alpha d(x, y)+\beta \frac{d(y, T y) d(x, T x)}{d(x, y)}
$$

for some $\alpha, \beta \geqslant 0$ such that $0<\alpha+\beta<1$. If $\mathrm{T}$ preserves order and there exists a starting point $x_{0} \in \mathrm{X}$, then $\mathrm{T}$ has a fixed point.

In the next results, we omit the continuity assumption of the operator $T$ and we replace it with $\preceq$ regularity condition of the partially ordered set $X$.

Theorem 2.13. Let $\mathrm{T}: \mathrm{X} \rightarrow \mathrm{X}$ be a generalized $\preceq-\theta$-contraction of type (I) for some $\theta \in \Theta_{c}$. Suppose that $\mathrm{T}$ preserves order. If $\mathrm{X}$ is $\preceq$-regular and there exists a starting point $\mathrm{x}_{0} \in \mathrm{X}$, then $\mathrm{T}$ has a fixed point.

Proof. Following the proof of Theorem 2.7, the sequence

$$
x_{n}=T^{n} x_{0} \text { for all } n
$$

is Cauchy in the complete metric space $(X, d)$ and it satisfies

$$
\left(x_{n}, x_{n+1}\right) \in E_{\preceq} \text { for all } n .
$$

Let $z \in X$ be such that $x_{n} \rightarrow z$ as $n \rightarrow \infty$. From the $\preceq$-regularity of the partially ordered set $X$, we have

$$
\left(x_{n}, z\right) \in E_{\preceq} \text { for all } n .
$$

Set

$$
\mathbb{I}_{z}=\left\{n: x_{n}=T z\right\}
$$

We distinguish two cases.

Case 1. If $\left|\mathbb{I}_{z}\right|<\infty$.

In this case, for $n$ large enough, we have $d\left(T x_{n}, T z\right) \neq 0$. Suppose that $z$ is not a fixed point of $T$. We have

$$
\theta\left(d\left(T^{n+1} x, T z\right)\right) \leqslant\left[\theta\left(M\left(T^{n} x, z\right)\right)\right]^{k} \text { for all } n,
$$

where

$$
M\left(T^{n} \chi, z\right)=\max \left\{d\left(T^{n} x, z\right), \frac{d(z, T z)\left(1+d\left(T^{n} x, T^{n+1} \chi\right)\right)}{1+d\left(T^{n} x, z\right)}\right\}
$$

Letting $n \rightarrow \infty$ in the above inequality and using the continuity of $\theta$, we obtain

$$
\theta(d(z, T z)) \leqslant[\theta(d(z, T z))]^{k}<\theta(d(z, T z)),
$$

which is a contradiction. Thus we proved that $z$ is a fixed point of T.

Case 2. If $\left|\mathbb{I}_{z}\right|=\infty$.

In this case, we can extract a subsequence $x_{n_{r}}$ of $\left\{x_{n}\right\}$ such that $x_{n_{r}} \subset \mathbb{I}_{z}$. This implies immediately (from the uniqueness of the limit) that $z=T z$.

So, in both cases, $z$ is a fixed point of $T$.

As consequences, we deduce the following results. 
Corollary 2.14. Suppose that $\mathrm{T}: \mathrm{X} \rightarrow \mathrm{X}$ is an operator satisfying the following condition: for all $(\mathrm{x}, \mathrm{y}) \in \mathrm{E}_{\preceq}$,

$$
d(T x, T y) \leqslant k \max \left\{d(x, y), \frac{d(y, T y)(1+d(x, T x))}{1+d(x, y)}\right\}
$$

for some $\mathrm{k} \in(0,1)$. If $\mathrm{T}$ preserves order, $\mathrm{X}$ is $\preceq$-regular, and there exists a starting point $\mathrm{x}_{0} \in \mathrm{X}$, then $\mathrm{T}$ has a fixed point.

Corollary 2.15. Suppose that $\mathrm{T}: \mathrm{X} \rightarrow \mathrm{X}$ is an operator satisfying the following condition: for all $(\mathrm{x}, \mathrm{y}) \in \mathrm{E}_{\preceq}$,

$$
d(T x, T y) \leqslant \alpha d(x, y)+\beta \frac{d(y, T y)(1+d(x, T x))}{1+d(x, y)}
$$

for some $\alpha, \beta \geqslant 0$ such that $0<\alpha+\beta<1$. If $\mathrm{T}$ preserves order, $\mathrm{X}$ is $\preceq$-regular, and there exists a starting point $x_{0} \in X$, then $\mathrm{T}$ has a fixed point.

Take $\beta=0$ in the above result, we obtain Nieto-Lopez fixed point theorem [22]. Proceeding as in the proof of Theorem 2.13, we obtain the following result.

Theorem 2.16. Let $\mathrm{T}: \mathrm{X} \rightarrow \mathrm{X}$ be a generalized $\preceq$ - $\theta$-contraction of type (II) for some $\theta \in \Theta_{\mathrm{c}}$. Suppose that $\mathrm{T}$ preserves order. If $\mathrm{X}$ is $\preceq$-regular and there exists a starting point $\mathrm{x}_{0} \in \mathrm{X}$, then $\mathrm{T}$ has a fixed point.

As consequences, we obtain the following results.

Corollary 2.17. Suppose that $\mathrm{T}: \mathrm{X} \rightarrow \mathrm{X}$ is an operator satisfying the following condition: for all $(\mathrm{x}, \mathrm{y}) \in \mathrm{E}_{\preceq}$ with $x \neq y$,

$$
d(T x, T y) \leqslant k \max \left\{d(x, y), \frac{d(y, T y) d(x, T x)}{d(x, y)}\right\}
$$

for some $\mathrm{k} \in(0,1)$. If $\mathrm{T}$ preserves order, $\mathrm{X}$ is $\preceq$-regular, and there exists a starting point $\mathrm{x}_{0} \in \mathrm{X}$, then $\mathrm{T}$ has a fixed point.

Corollary 2.18. Suppose that $\mathrm{T}: \mathrm{X} \rightarrow \mathrm{X}$ is an operator satisfying the following condition: for all $(\mathrm{x}, \mathrm{y}) \in \mathrm{E}_{\preceq}$ with $x \neq y$,

$$
d(T x, T y) \leqslant \alpha d(x, y)+\beta \frac{d(y, T y) d(x, T x)}{d(x, y)}
$$

for some $\alpha, \beta \geqslant 0$ such that $0<\alpha+\beta<1$. If $\mathrm{T}$ preserves order, $\mathrm{X}$ is $\preceq$-regular, and there exists a starting point $\mathrm{x}_{0} \in \mathrm{X}$, then $\mathrm{T}$ has a fixed point.

Remark 2.19. All the above results hold if we replace the set $\mathrm{E}_{\preceq}$ by any set $\mathrm{H} \subseteq \mathrm{X} \times \mathrm{X}$. In this case, we say that $\mathrm{T}: \mathrm{X} \rightarrow \mathrm{X}$ is H-stable if

$$
(x, y) \in H \Rightarrow(T x, T y) \in H .
$$

We say that $x_{0} \in X$ is a starting point if $\left(x_{0}, T x_{0}\right) \in H$.

We say that $X$ is $H$-regular if for every sequence $x_{n} \subset X$ such that $\left(x_{n}, x_{n+1}\right) \in H$ for every $n$, we have

$$
x_{n} \rightarrow x \text { as } n \rightarrow \infty \Rightarrow\left(x_{n}, x\right) \in H \text { for every } n .
$$

We say that $T$ is a generalized $H-\theta$-contraction of type (I) for some $\theta \in \Theta$, if it satisfies the following condition:

$$
(x, y) \in H, d(T x, T y) \neq 0 \Longrightarrow \theta(d(T x, T y)) \leqslant[\theta(M(x, y))]^{k}
$$

for some $k \in(0,1)$.

We say that $T$ is a generalized $H$ - $\theta$-contraction of type (II) for some $\theta \in \Theta$, if it satisfies the following condition:

$$
(x, y) \in H, d(T x, T y) \neq 0 \Longrightarrow \theta(d(T x, T y)) \leqslant[\theta(N(x, y))]^{k}
$$


for some $k \in(0,1)$.

Observe that in the particular case, where $\mathrm{H}=\mathrm{E}_{\preceq}$, an $\mathrm{H}$-stable operator is an operator that preserves order. An $\mathrm{H}$-regular set $X$ is a $\preceq$-regular set. A generalized $\mathrm{H}-\theta$-contraction of type (I) is a generalized $\preceq-\theta$-contraction of type (I). A generalized $\mathrm{H}$ - $\theta$-contraction of type (II) is a generalized $\preceq$ - $\theta$-contraction of type (II).

Using the above remark, we can deduce the following fixed point theorems for cyclic $\theta$-contractions that generalize the fixed point theorem of Kirk et al. [16].

Theorem 2.20. Suppose there exist two nonempty closed subsets $\mathrm{A}$ and $\mathrm{B}$ of $\mathrm{X}$ such that the mapping $\mathrm{T}: \mathrm{A} \cup \mathrm{B} \rightarrow$ $A \cup B$ satisfies the following conditions:

(i) $\mathrm{T}(\mathrm{A}) \subseteq \mathrm{B}$ and $\mathrm{T}(\mathrm{B}) \subseteq \mathrm{A}$;

(ii) for all $(x, y) \in(A \times B) \cup(B \times A)$,

$$
d(T x, T y) \neq 0 \Longrightarrow \theta(d(T x, T y)) \leqslant[\theta(M(x, y))]^{k}
$$

for some $\theta \in \Theta_{c}$ and $k \in(0,1)$.

Then $\mathrm{T}$ has a fixed point $z \in A \cap B$.

Proof. Take $\mathrm{H}=(\mathrm{A} \times \mathrm{B}) \cup(\mathrm{B} \times \mathrm{A})$. Observe that $\mathrm{T}$ is $\mathrm{H}$-stable from the condition (i). From (ii), $\mathrm{T}$ is a generalized $H-\theta$-contraction of type (I). On the other hand, since $A$ and $B$ are closed, the set $A \cup B$ is H-regular. Also, any point $x_{0} \in A \cup B$ is a starting point from the condition (i). By Theorem 2.13, $T$ has a fixed point $z \in A \cup B$. Finally, condition (i) yields $z \in A \cap B$.

Similarly, we can establish the following result.

Theorem 2.21. Suppose there exist two nonempty closed subsets $\mathrm{A}$ and $\mathrm{B}$ of $\mathrm{X}$ such that the mapping $\mathrm{T}: \mathrm{A} \cup \mathrm{B} \rightarrow$ $A \cup B$ satisfies the following conditions:

(i) $\mathrm{T}(\mathrm{A}) \subseteq \mathrm{B}$ and $\mathrm{T}(\mathrm{B}) \subseteq \mathrm{A}$;

(ii) for all $(x, y) \in(A \times B) \cup(B \times A)$,

$$
d(T x, T y) \neq 0 \Longrightarrow \theta(d(T x, T y)) \leqslant[\theta(N(x, y))]^{k}
$$

for some $\theta \in \Theta_{c}$ and $k \in(0,1)$.

Then $\mathrm{T}$ has a fixed point $z \in A \cap \mathrm{B}$.

\section{Some open questions}

In Theorem 2.1, we supposed that $\theta$ is continuous. Is it possible to omit this assumption without the continuity of T? We have the same question for Theorem 2.4.

\section{Acknowledgment}

The second author extends his appreciation to the Deanship of Scientific Research at King Saud University for funding this work through research group No RGP-237.

\section{References}

[1] S. Banach, Sur les opérations dans les ensembles abstraits et leur applications aux équations intégrales, Fundam. Math., 3 (1922), 133-181. 1, 1, 2

[2] M. Berinde, V. Berinde, On a general class of multi-valued weakly Picard mappings, J. Math. Anal. Appl., 326 (2007), 772-782. 1

[3] D. W. Boyd, J. S. W. Wong, On nonlinear contractions, Proc. Amer. Math. Soc., 20 (1969), 458-464. 1

[4] A. Branciari, A fixed point theorem of Banach-Caccioppoli type on a class of generalized metric spaces, Publ. Math. Debrecen, 57 (2000), 31-37.1 
[5] M. Cherichi, B. Samet, Fixed point theorems on ordered gauge spaces with applications to nonlinear integral equations, Fixed Point Theory Appl., 2012 (2012), 19 pages. 1

[6] L. Cirić, A generalization of Banach's contraction principle, Proc. Amer. Math. Soc., 45 (1974), 267-273. 1

[7] L. Cirić, Multi-valued nonlinear contraction mappings, Nonlinear Anal., 71 (2009), 2716-2723. 1

[8] B. K. Dass, S. Gupta, An extension of Banach contraction principle through rational expression, Indian J. Pure Appl. Math., 6 (1975), 1455-1458. 1, 1, 2

[9] M. Frigon, Fixed point results for generalized contractions in gauge spaces and applications, Proc. Amer. Math. Soc., 128 (2000), 2957-2965. 1

[10] D. S. Jaggi, Some unique fixed point theorems, Indian J. Pure Appl. Math., 8 (1977), 223-230. 1, 1, 2

[11] S. Jankovic, Z. Kadelburg, S. Radenović, On cone metric spaces: a survey, Nonlinear Anal., 74 (2011), 2591-2601. 1

[12] M. Jleli, E. Karapinar, B. Samet, Further generalizations of the Banach contraction principle, J. Inequal Appl., 2014 (2014), 9 pages. 1, 1

[13] M. Jleli, B. Samet, A new generalization of the Banach contraction principle, J. Ineq. Appl., 2014 (2014), 8 pages. 1

[14] E. Karapinar, Fixed points results for $\alpha$ admissible mapping of integral type on generalized metric spaces, Abstr. Appl. Anal., 2015 (2015), 11 pages. 1

[15] W. A. Kirk, N. Shahzad, Generalized metrics and Caristis theorem, Fixed Point Theory Appl., 2013 (2013), 9 pages. 1

[16] W. A. Kirk, P. S. Srinivasan, P. Veeramani, Fixed points for mappings satisfying cyclical contractive conditions, Fixed Point Theory, 4 (2003), 79-89. 1, 2

[17] J. T. Markin, A fixed point theorem for set-valued mappings, Bull. Amer. Math. Soc., 74 (1968), 639-640. 1

[18] S. G. Mathews, Partial metric topology, Papers on general topology and applications, New York, (1994). 1

[19] A. Meir, E. Keeler, A theorem on contraction mappings, J. Math. Anal. Appl., 28 (1969), 326-329. 1

[20] N. Mizoguchi, W. Takahashi, Fixed point theorems for multivalued mappings on complete metric spaces, J. Math. Anal. Appl., 141 (1989), 177-188. 1

[21] S. B. Nadler, Multi-valued contraction mappings, Pacific J. Math., 30 (1969), 475-488. 1

[22] J. J. Nieto, R. Rodríguez-López, Contractive mapping theorems in partially ordered sets and applications to ordinary differential equations, Order, 22 (2005), 223-239. 1, 2

[23] E. Rakotch, A note on contractive mappings, Proc. Amer. Math. Soc., 13 (1962), 459-465. 1

[24] A. C. M. Ran, M. C. B. Reurings, A fixed point theorem in partially ordered sets and some applications to matrix equations, Proc. Amer. Math. Soc., 132 (2004), 1435-1443. 1, 2

[25] S. Reich, Fixed points of contractive functions, Boll. Un. Mat. Ital., 5 (1972), 26-42. 1

[26] T. Suzuki, Fixed point theorem for asymptotic contractions of Meir-Keeler type in complete metric spaces, Nonlinear Anal., 64 (2006), 971-978. 1

[27] E. Tarafdar, An approach to fixed point theorems on uniform spaces, Trans. Amer. Math. Soc., 191 (1974), $209-225.1$

[28] D. Wardowski, Fixed points of a new type of contractive mappings in complete metric spaces, Fixed Point Theory Appl., 2012 (2012), 6 pages. 1

[29] C.-K. Zhang, J. Zhu, P.-H. Zhao, An extension of multi-valued contraction mappings and fixed points, Proc. Amer. Math. Soc., 128 (2000), 2439-2444. 1 